\title{
Do blood-borne calcifying nanoparticles self-propagate?
}

\author{
Grace Mathew' \\ David S McKay ${ }^{2}$ \\ Neva Çiftçioglu² \\ 'Nanobac Pharmaceuticals Inc, \\ Johnson Space Center, Houston, TX, \\ USA; ${ }^{2}$ NASA Johnson Space Center, \\ Houston, TX, USA
}

\begin{abstract}
The nanotechnology industry is currently in the process of producing new nanoparticles. The biological activity of nanoparticles including adverse as well as beneficial effects tends to increase as their size decreases. The smaller the particles are, the greater their bioactivity and toxicity. Thus, one can easily conjecture the impact of a nanoparticle if it could also self-replicate. This in vitro study reveals the self-propagating ability of unique calcifying nanoparticles (CNP) that can be as small as $50 \mathrm{~nm}$ in size and found in blood, blood products, and calcified soft tissues. Although specific detection techniques, morphological characteristics and biomineralizing properties of CNP are well established, their genomic information and selfpropagating capability have always been challenged. The objective of this study is to document the propagation of CNP under physiological conditions, using inverted light microscopy (LM) and the Biostation IM time-lapse imaging system. Their detailed morphological structure was examined using scanning (SEM) and transmission (TEM) electron microscopy. This present study, in conjunction with previous findings of metabolic activity, antibiotic sensitivity, antibody specificity, morphological aspects and infectivity, validates CNP as self-replicators. Therefore these sterile-filterable, blood-borne nanoparticles should be of concern to the nanomedicine industry.
\end{abstract}

Keywords: calcifying nanoparticles, time-lapse photography, self-replication, apatite

\section{Introduction}

Nanotechnology is a rapidly evolving discipline that has aroused the interest of both the scientific community and the public. Ordinary materials such as carbon or silicon, when reduced to nanoscale level, often exhibit novel and unpredictable characteristics such as extraordinary strength, resistance and chemical reactivity that the same material does not possess at the micro or macro-scale (Nel et al 2004). Although nanotechnology industry is very successful in manufacturing a variety of nanoparticles, they have yet to be successful in developing methods to eliminate them. Currently, sterile filtration is the most commonly used technique for biopharmaceutical products although it has no satisfactory capability of removing particles below $100 \mathrm{~nm}$ in size (Çiftçioglu et al 1997a).

The discovery of calcifying nanoparticles (CNP) in blood and blood products in the early 1990s resulted in a breakthrough in the field of biology. CNP have been shown to be bacteria-like, pleomorphic, infectious particles that possess unique properties including the capability of passing sterilization filters because of their small size, and resistance to heat and $\gamma$-irradiation at doses typically fatal for conventional bacteria (Çiftçioglu et al 1997a, 1997b, 2007; Kajander et al 1997; Kajander and Çiftçioglu 1998; Miller-Hjelle et al 2003). They deposit calcium and phosphate as an envelope at physiologic $\mathrm{pH}$ and mineral concentrations (Kajander and Çiftçioglu 1998; MillerHjelle et al 2003). CNP has been associated with diverse calcification-related health problems such as arteriosclerosis, kidney stone, gall stone, dental pulp stone, prostatitis, 
Alzheimer's, polycystic kidney, and cancer (Çiftçioglu et al 1998a, 1998b; Kajander et al 1999; Kajander et al 2001; Puskas et al 2005; Altundag et al 2006; Shiekh et al 2006; Wang et al 2006; Wood and Shoskes 2006). CNP exert cytotoxic effects on some mammalian cells in vitro and in living organisms in vivo (Akerman et al 1997; Çiftçioglu and Kajander 1998).

Despite their potential role in major medical health problems (Kajander 2006), CNP could not be categorized under any group of infectious agents because of their unusual properties and lack of genomic information. Also, the selfreplicating nature of CNP has often been questioned (Cisar et al 2000). The terms "self-replication", "self-assembly", or "self-propagation" have been widely used for all systems including nanomachines, crystals, computer viruses, and memes (Freitas and Merkle 2004). Here we have investigated the self-replicating ability of these medically important nanoparticles.

CNP have morphological changes in different culture conditions. For example they form excessive amounts of biofilm when stressed with antibiotics, calcify less when cultured in microgravity conditions and calcify profusely when serum/protein concentration is reduced below 5\% (Bjorklund et al 1998; Kajander et al 2003; Çiftçioglu et al 2005). However their antigenicity remains the same, and they are still recognized by CNP-specific monoclonal antibodies (mAb) (Kajander et al 2003). Figure 1 shows a schematic diagram of the growth phases of CNP, cultured with and without serum containing medium. Starting at Stage A, in a serum containing medium, the tiny cell-like coccobacillar forms from the stock culture beginning to acquire thin coatings of apatite crystals on their organic membrane. They grow slightly larger in size as a result of this apatite accumulation (Stage B) and form dumbbell-shaped forms (Stage C). These forms can be passaged at 1/10 dilution for years and they continue to reproduce, maintaining the same shape and narrow size range (50-400 nm, Figure 1 i). The cycle from stages A to C and C to A can continue indefinitely (Vali et al 2001). These small, coccobacillar CNP are referred to as serum-CNP (S-CNP) and this type of CNP is cultured when Dulbecco's Modified Eagle's Medium (DMEM) is supplemented with 10\% fetal bovine serum (FBS). However, if these S-CNP are passaged into serum (protein)-free media, the protein depletion causes CNP to produce biofilm-like material and they attach to the surface of the culture vessel where they develop several apatite mineral layers around them (Figure 1 ii) forming "igloos" or "shells" (Figure 1 stages D-G, ii and iii) (Kajander et al 2003). These igloo forms harbor in their interior many S-CNP in a semi-dormant state, which can be observed only by using electron microscopy techniques (Çiftçioglu et al 1997b) (Figure $1 \mathrm{iv}$ ). We refer to these attached CNP igloos as serumfree CNP (SF-CNP). We have SF-CNP cultures that have been passaged at 1/10 dilution monthly for over 17 years in serum and protein-free DMEM. So, the cycle from stages D to F and F to D can continue indefinitely (as schematized in Figure 1). SF-CNP mineralize and grow larger in size (1-10 $\mu \mathrm{m})$ when compared with S-CNP, as a result of calcium and phosphate deposition on their surface as shown in Figure 1 D-G, and ii-iv (Miller-Hjelle et al 2003).

The addition of the serum to the culture media brings the system back to Stage A. Many proteinaceous inhibitors of apatite crystal formation have been identified in serum, which may account for the observed dissolution of the mineral layers (Garnett and Dieppe 1990). The SEM image in Figure 1 iv, and LM images in Figure 1: G1-G5 show how SF-CNP detaches from the surface and the apatite layers dissolve to release the S-CNP (50-100 nm) when serum/protein replenishment takes place.

The objective of this study is to document the propagation of both types of CNP under physiological conditions, using inverted LM and the BioStation IM time-lapse imaging system. While this optical microscopic imaging may seem like a simple technology, it is the only available technique of today for viewing the nanometersized particles in real time.

\section{Materials and methods CNP cultures}

In all experimental analyses we used the same CNP which was isolated from FBS (Sera Lab, Lot: 901045, Country; England), and deposited in German Bank DSM no. 5819-5821. Two types of CNP (as described in Figure 1) were examined for the observation of self-propagation in specific culture conditions; subcultures of SF-CNP were conducted under strict aseptic conditions by passing a small inoculum (1/10 of a 3 week old culture) of SF-CNP into culture flasks with fresh DMEM (Invitrogen, Carlsbad, CA, USA; supplemented with L-glutamine) under cell culture conditions $\left(37^{\circ} \mathrm{C} ; 5 \%-10 \% \mathrm{CO}_{2} ; 95 \%\right.$ air at $90 \%$ humidity). For observation of S-CNP, the culture was passaged 1/50 into FBS-free DMEM. Therefore the final serum concentration was reduced to $0.3 \%$ which caused S-CNP to attach to the culture vessel and make microscopic observations possible. As negative controls, DMEM with and without FBS (0.3\%), and also inorganic hydroxyapatite crystals (Sigma-Aldrich, St Louis, MO, USA) in the two media, by omitting the 

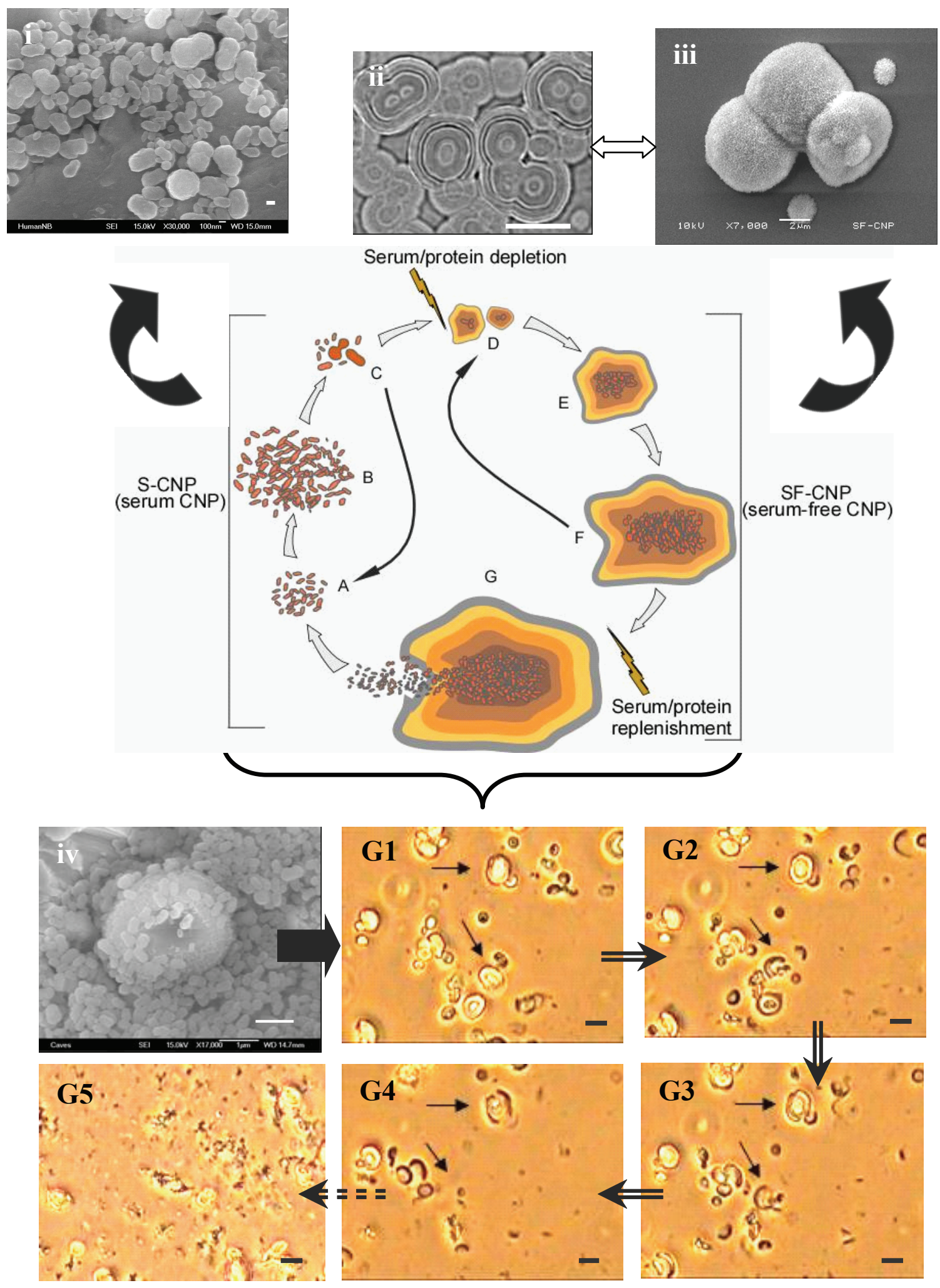

Figure I Schematic diagram of the growth phases of CNP (cross-sectional view) cultured with and without FBS with corresponding SEM and LM images, and LM observation of dissolution of SF-CNP to S-CNP.A, B, and C are the forms of CNP in the presence of serum (S-CNP). (i): SEM image of S-CNP.D, E, and F are the forms of CNP in the absence of FBS (SF-CNP). (ii): LM image of apatite layers of SF-CNP. (iii): SEM image of the formation shown in (ii). G: Schema of SF-CNP when exposed to FBS, apatite layers dissolve releasing the S-CNP within 24 h. (iv): SEM image of phase G. GI-G5:A series of LM images showing the dissolution of SF-CNP apatite layers and clumps of S-CNP released, with the replenishment of FBS. Bars: (i) $=100 \mathrm{~nm}$; (ii) $=5 \mu \mathrm{m}$; (iii) $=2 \mu \mathrm{m}$; (iv) $=1 \mu \mathrm{m}$; (GI-G5) $=5 \mu \mathrm{m}$.

Abbreviations: CNP, calcifying nanoparticles; FBS, fetal bovine serum; LM, light microscopy; SEM, scanning electron microscopy. 
CNP addition step were incubated under the same culture conditions and culture period. All cultures were observed with microscopy for 3 weeks and were not re-fed with fresh medium for the entire duration of the experiment. At the end of experiment, cultures were passed through quality control tests checking for conventional bacteria contamination, and CNP epitope positivity using the double staining technique as described earlier (Kajander and Çiftçioglu 1998). The Double staining technique is a combination technique of immunofluorescence staining with CNP-specific mAb 8D10 (Nanobac, OY), and Hoechst (\#33258) fluorochrome staining (Kajander et al 2003).

\section{Microscopy and photography}

Two types of imaging were performed: a) using the conventional inverted LM (Nikon, Eclipse TE2000-U) in the phase contrast mode and b) imaging using Nikon's BioStation IM (Nikon Instruments Inc., Melville, NY).

For observation of SF-CNP replication with the conventional LM, objectives with $20 \mathrm{X}$ and $60 \mathrm{X}$, eyepiece with 10X, and an intermediate optics of 1.5X magnifications were used. The culture flasks were indexed with a diamond pen so as to view the same field. Each time the same focus planes were located using the 2 magnifications: $300 \mathrm{X}$ and 900X. Images were captured digitally using Nikon's charged-coupled device camera (Digital Sight DS-L1). A few large CNP were marked 1-4 (with arrows) on the images (Figure 2) in order to identify the same spots throughout the observation.

BioStation IM is a time-lapse imaging system on loan to us from Nikon, so we only had time for a limited set of time-lapse experiments. This microscopy system is a novel compact cell incubation and monitoring system allowing time-lapse cell imaging without the set-up and alignment complexity of conventional time-lapse imaging systems. The system combines an incubator that maintains mammalian cell culture conditions, a microscope with a numerical aperture of NA 0.80 , delivering high resolution images in phase-contrast mode, an internal motorized stage supporting $\mathrm{X}, \mathrm{Y}$, and $\mathrm{Z}$ dimensional movement with reduced focus-drift, and a high performance CCD digital imaging camera for capturing timelapse image sequences (see http://www.nikonusa.com). Both S-CNP and SF-CNP were monitored in $30 \mathrm{~mm}$ cover-slip bottom petri-dishes under $40 \mathrm{X}$ magnification. Time-lapse imaging was conducted for 5 days with images taken at regular intervals. The exposure time was $1 / 10$ sec, at $1600 \times$ 1200 pixel resolution. Both S-CNP and SF-CNP counts were performed using ImageJ software (NIH, USA).
For SEM, at the end of the experiments, the cultures were either scraped with a cell culture scraper, harvested by centrifugation (Mikro22R, Hettich, Germany) at 14,000 g for $20 \mathrm{~min}$, and using the pellet as a sample, or a piece of culture vessel having attached CNP was cut with a heated scalpel and used in the sample preparation. The samples were washed twice with phosphate buffered saline, $\mathrm{pH} 7.4$ (PBS) and fixed with $2 \%$ gluteraldehyde in PBS for $16 \mathrm{~h}$ at $4{ }^{\circ} \mathrm{C}$. The fixed samples were washed twice with PBS, dehydrated with gradually increasing ethanol concentrations, and dried with a critical point dryer (Çiftçioglu et al 2002). The samples were coated with gold (thickness, 20 to $40 \mathrm{~nm}$ ) prior to examination with a JEOL 5910LV SEM (Jeol Ltd., Tokyo, Japan) with attached energy dispersive spectroscopy (EDS).

For TEM, SF-CNP cultures were harvested, fixed with formaldehyde-gluteraldehyde mixture, epoxy-embedded, and sectioned as described earlier (Kajander and Çiftçioglu 1998). For S-CNP cultures, negative staining is applied and observed under TEM (JEOL 2000FX, Tokyo, Japan) (Çiftçioglu and Kajander 1998).

\section{Results}

For our initial set of experiments we used only SF-CNP. Figure 2 shows optical microscopic images of culture follow up of SF-CNP over a period of 25 days. Coccoidal particles $(\leq 1 \mu \mathrm{m})$ were observed which attached to the culture vessels by the end of first day incubation. These particles are the S-CNP released from the main culture during passaging. Figure 3 shows igloo-shaped SF-CNP by SEM and TEM after they are detached from the culture flask. On the first day of the SF-CNP culture, both small- and larger-sized, igloo-shaped formations were observed in small number. Appearance of small particles around the large coccoid cells is seen from Day 2 (Figure 2B). Over time the particles become more visible, optically opaque, and bigger due to apatite deposition. In this experiment, we observed that CNP grow within a size range between $0.5-6 \mu \mathrm{m}$ and apparently increase in number.

Using Nikon's BioStation IM imaging system, both SF and S-CNP were imaged for a period of 5 days each. Although this culture period is short, we see an increase in number in both types of CNP (Figures 4 and 5). The results obtained were comparable with that from the previous experiment using inverted LM. A graph of SF-CNP count against time was plotted (Figure 4D). The total culture period was $120 \mathrm{~h}$. The graph indicates a linear increase in SF-CNP number with time. Time-lapse imaging of S-CNP shows a gradual increase in their number over a period of 


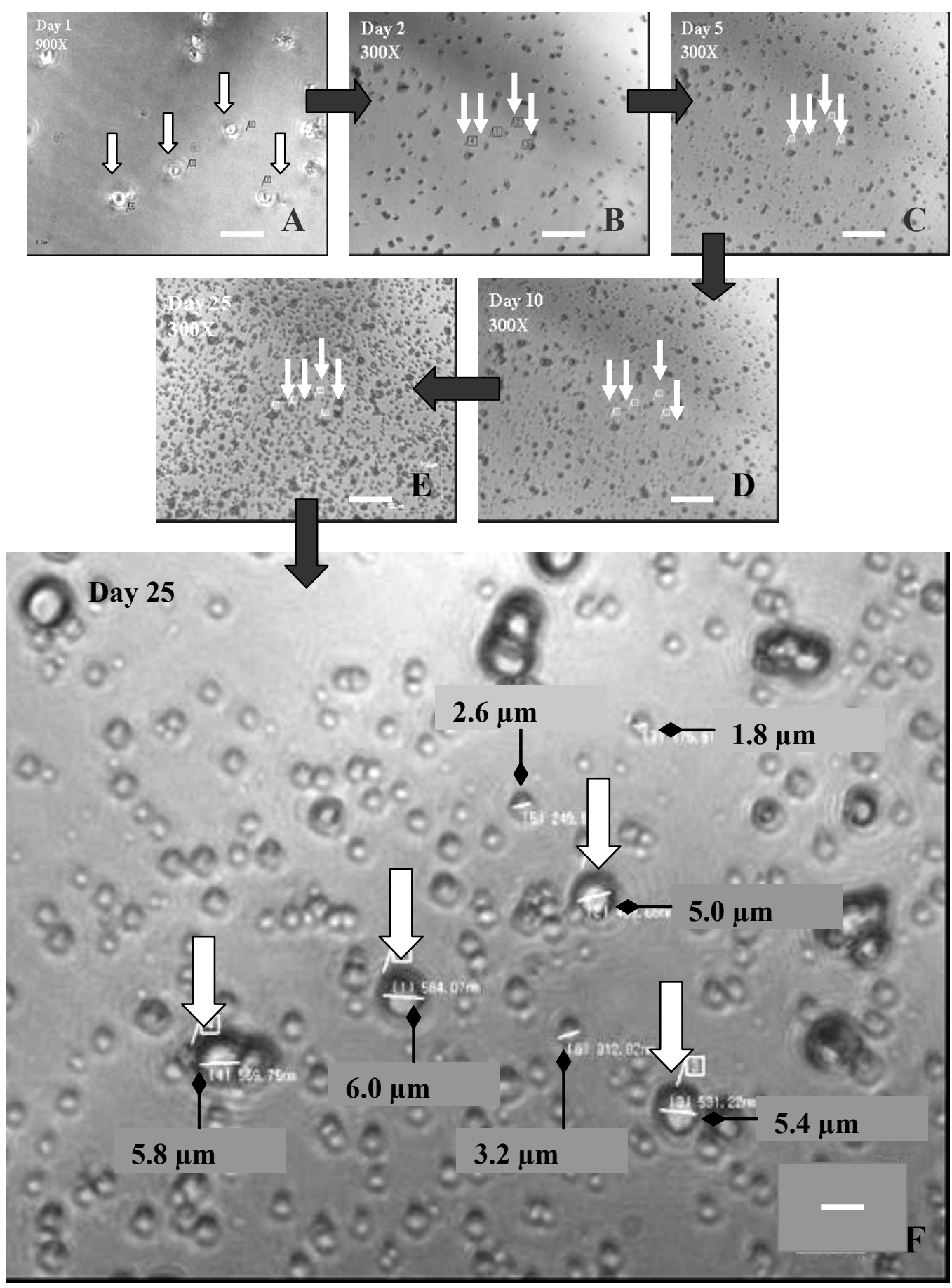

Figure 2 Optical micrograph of SF-CNP showing an increase in number over a culture period of 25 days. (A) Day I at 900X magnification; (B-E) Days 2, 5, 10, and 25, respectively at 300X magnification. The white arrows in each image indicate the same large SF-CNP on the same spot throughout the experiment. (F) measurements of a few SF-CNP on Day 25 at 900X magnification. All particles seen in the images are the different sizes of SF-CNP. Bars: $(\mathbf{A})=15 \mu \mathrm{m} ;(\mathbf{B}),(\mathbf{C}),(\mathbf{D})$, and $(\mathbf{E})=30 \mu \mathrm{m} ;(\mathbf{F})=5 \mu \mathrm{m}$. Abbreviation: SF-CNP, serum-free calcifying nanoparticles.

5 days (Figure 5). Preliminary studies over shorter time periods showed that replication was reproducible between runs (data not shown). For all the experiments conducted, negative controls without any CNP in DMEM, with and without FBS, did not show any particle formation or growth (data not shown). EDS showed Ca and P peaks in both forms of CNP (Figure 6). Also, negative controls with inorganic hydroxyapatite in similar media and culture conditions, did not show any increase in the apatite particle number or size (data not shown).

Interestingly, we observed similar propagation characteristics in both S-CNP and SF-CNP. The results of TEM and 


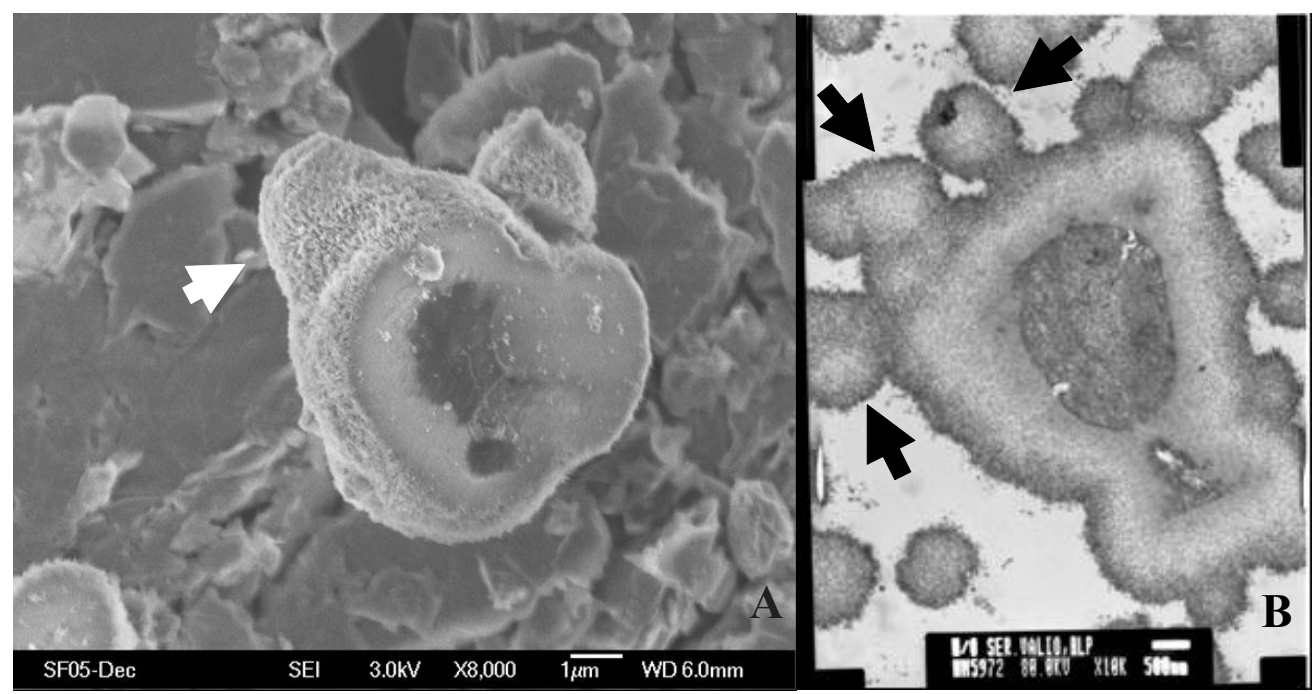

Figure 3 Electron microscopic images of SF-CNP. (A) SEM of an empty apatite "igloo" detached from the culture medium. (B) TEM section of a similar SF-CNP and its inner structure.Arrows point to the apparently budding side of the shell. Bars: $(\mathbf{A})=1 \mu \mathrm{m} ;(\mathbf{B})=500 \mathrm{~nm}$.

Abbreviations: SEM, scanning electron microscopy, SF-CNP, serum-free calcifying nanoparticles; TEM, transmission electron microscopy.

SEM analyses of both types of CNP showed spherical and semi-spherical particles with rough surface and budding-like structures (Figures 3 and 7) despite their difference in size and thickness of apatite envelope.

\section{Discussion}

In nanotechnology, in all nanoparticle production processes and applications, there is potential for exposure. Current methods for quality certification of high efficiency particulate air (HEPA) filters, respirator filters, and filters for biological processes do not routinely require testing particle sizes below $100 \mathrm{~nm}$. CNP is a good example of a blood-borne agent capable of passing filtration barriers with their exceptionally small size.

Critics have proposed CNP to be protein or crystal precipitates rather than a self-propagating agent (Cisar et al 2000; Vali et al 2001). To eliminate this possibility we conducted our study with serum-free culture media under physiological conditions. Also, the absence of serum in medium allowed the CNP to attach to the surface of the culture dish, facilitating their observation with LM over a prolonged time period. In our experiments, as negative controls, we used culture media with and without FBS $(0.3 \%)$ and incubated them in the absence of CNP under similar culture conditions and culture period as CNP cultures. We have not observed any protein precipitation or crystal formation in the negative controls (data not shown).

In previous studies, the S-CNP doubling rate has been calculated as a mean value of 72 hours with a logarithmic increase in turbidity in cultures with lag and log growth phases (Çiftçioglu et al 1997b). In this study, since the S-CNP were passaged into SF media, they attached themselves to the culture dish. Hence, measurement of turbidometric changes for these attached CNP was not possible. It has also been reported that for SF-CNP the growth rate is even slower (Çiftçioglu et al 1997b).

Previous time-lapse studies of crystal growth have shown sedimentation of microcrystals onto the larger crystals with formation of defects (Malkin et al 1996; Piana et al 2005). Also, for crystal growth to occur, supersaturated conditions are required at least during preliminary stages (Goe 1976; Fosythe et al 1994; Vali et al 2001). CNP self propagates under physiological conditions. For more than a decade, CNP have been cultured and passaged under physiological conditions similar to mammalian culture conditions, without any change in their growth characteristics and specific monoclonal antibody recognizing epitopes (Çiftçioglu et al 2006). Inorganic apatite under similar conditions, do not show any growth or other characteristics as shown in Table 1.

The exact mechanism by which apatite is nucleated and formed around CNP is unknown. However, when serum or protein concentration is reduced $(\leq 5 \%)$ in the CNP culture, they start to mineralize and grow larger in size, forming "igloo" like structures due to calcium and phosphate deposition on their surface as confirmed by EDS analysis (Figure 6). As seen in SEM Figure 1-iii, these igloos can reach up to several microns thick, sheltering within them tiny nanometer-sized CNP. Within their apatite shelter, CNP continue to 

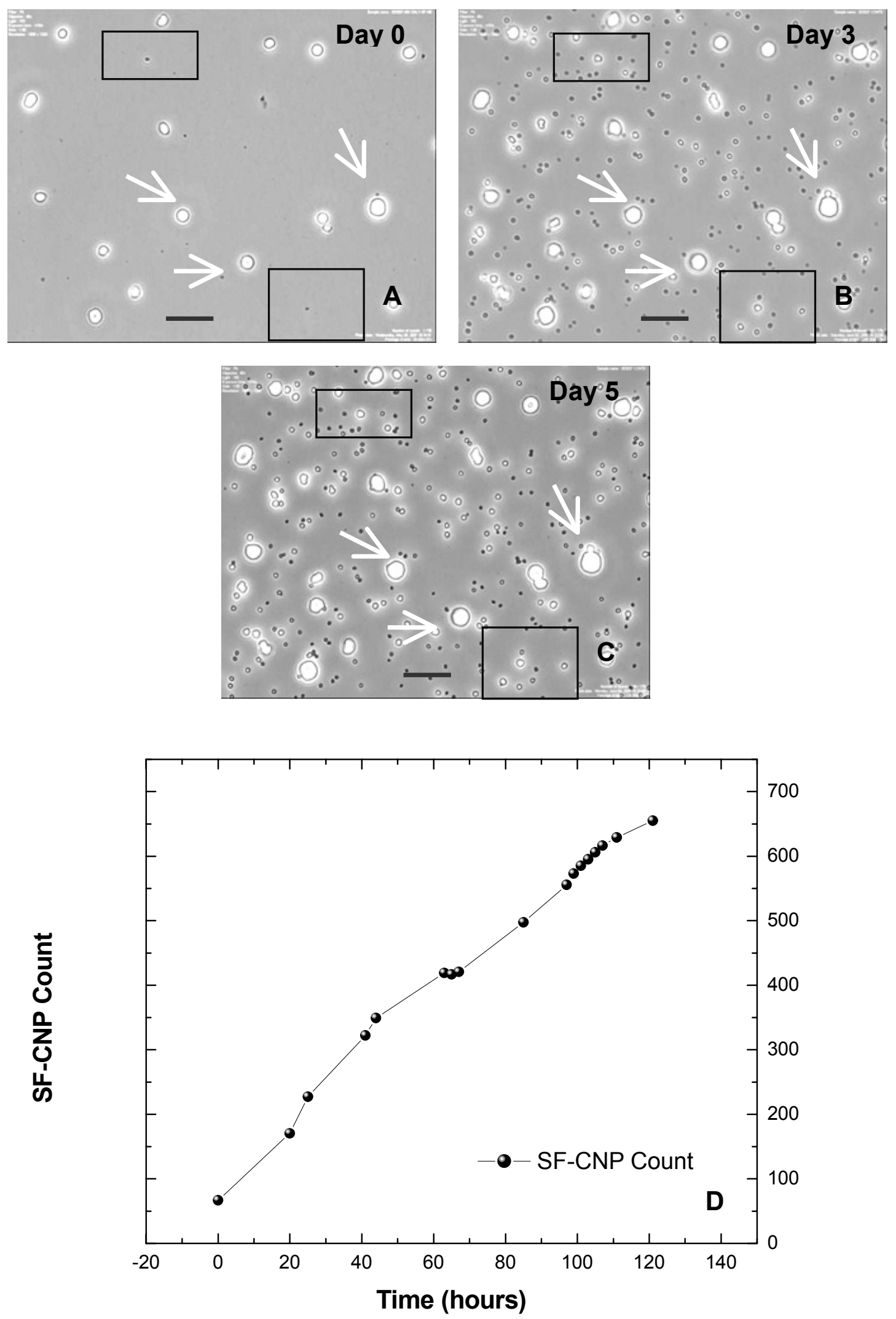

Figure 4 Time-lapse imaging and plot of SF-CNP from Day 0 to Day 5 using Nikon's BioStation IM. Only a few intermediate images of SF-CNP on Days 0,3 , and 5 at $40 \mathrm{X}$ magnification are shown. The white arrows mark some large SF-CNP on the same spot throughout the experiment. Note the small SF-CNP within the square blocks showing an increase in size and number over time.A graph of SF-CNP count against time in hours shows a linear increase in the SF-CNP number.The images and graph together imply an increase in size and number of SF-CNP over a period of 5 days. Bars: (A), (B), and $(\mathbf{C})=15 \mu \mathrm{m}$. Abbreviation: SF-CNP, serum-free calcifying nanoparticles. 

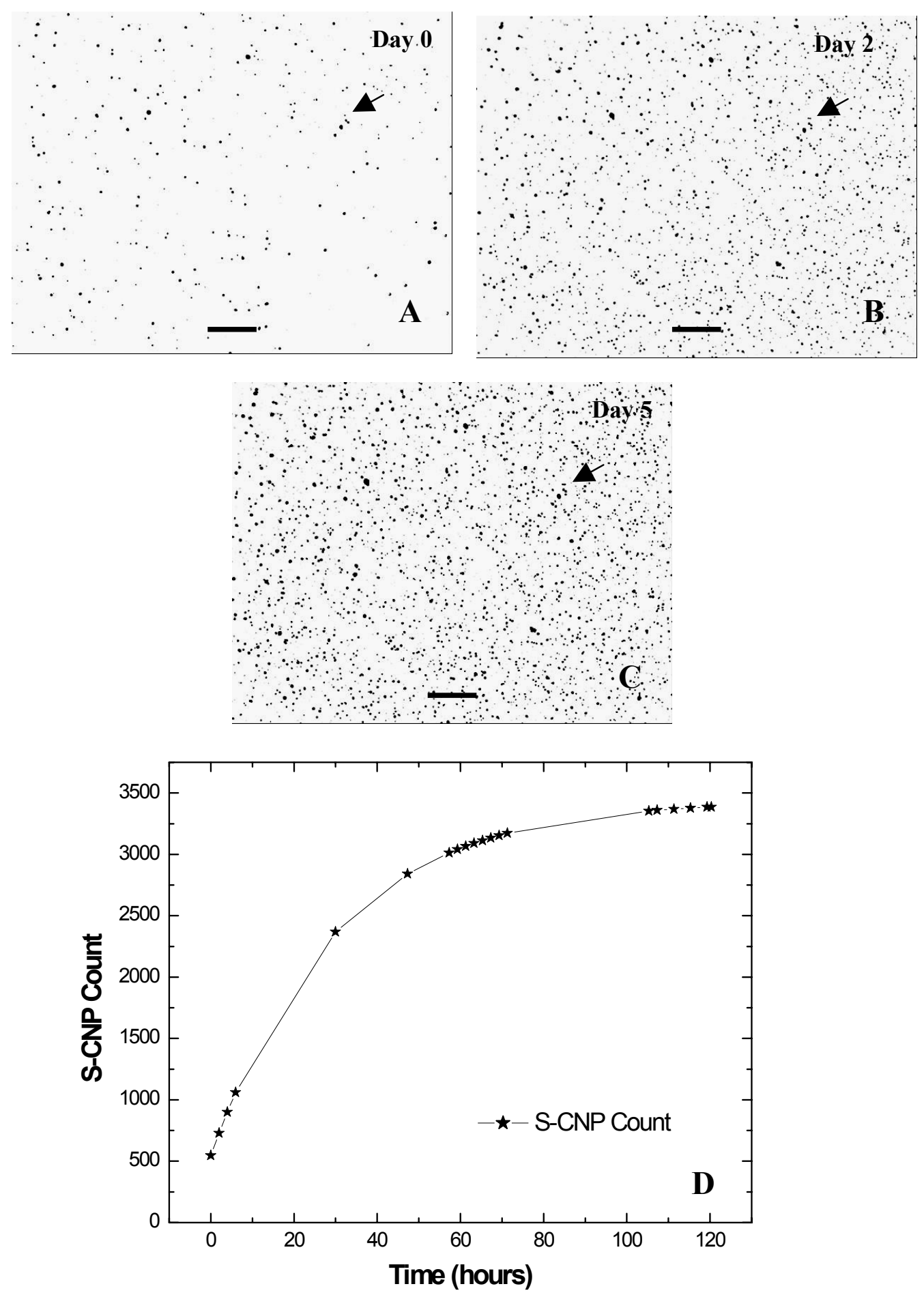

Figure 5 Time-lapse imaging and plot of S-CNP from Day 0 to Day 5 using Nikon's BioStation IM. Only a few intermediate images of S-CNP on Days 0,2 , and 5 at 40 X magnification are shown. The black arrows point to some S-CNP on the same spot throughout the experiment. Bars: $(\mathbf{A}),(\mathbf{B})$, and $(\mathbf{C})=15 \mu \mathrm{m}$. Abbreviation: S-CNP, serum calcifying nanoparticles.

grow but at a slower rate, in a semi-dormant state, and are released from their igloos when serum/protein is replenished (Figure 1-iv). This characteristic of CNP enables them to survive in body fluids as free nanometer-sized particles and also as thick plaques in calcified soft tissues.
Binary fission is the usual form of reproduction by bacteria, although a few bacteria and some eukaryotes (including yeasts) replicate by budding (Angert 2005). In environmental microbiology, it is known that there are aquatic microorganisms that use both binary division and budding mechanism while 


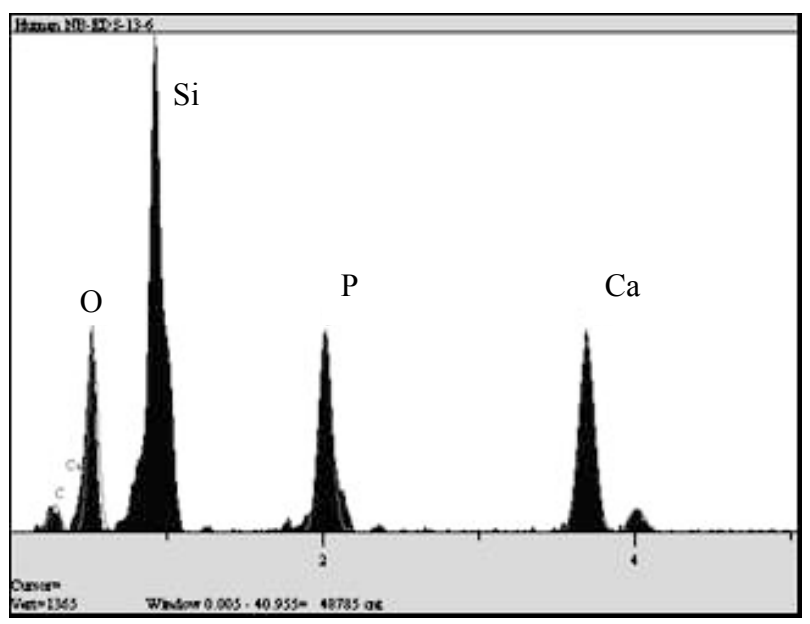

Figure 6 EDS analysis of CNP apatite. Si peak is because of the glass substrate on which CNP samples were cultured.

Abbreviations: CNP, calcifying nanoparticles; EDS, energy dispersive spectroscopy.

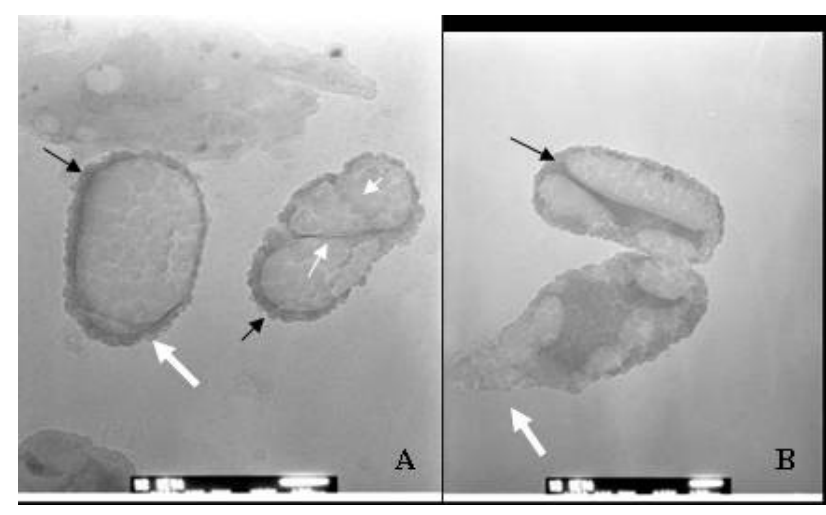

Figure 7 TEM image of "dividing" S-CNP. (A) Unique septa-like divisions ("cells" on the right, with white-small arrows). (B) A unique inner structure of CNP during division. Thick white arrows show budding-like formations, black arrows show dark stained apatite layer on the surface of CNP. Bars: $50 \mathrm{~nm}$.

Abbreviations: CNP, calcifying nanoparticles; S-CNP, serum calcifying nanoparticles; TEM, transmission electron microscopy.

Table I Comparison of two self-replicators: CNP and inorganic apatite crystals

\begin{tabular}{lll}
\hline Properties & CNP & Inorganic apatite crystals \\
\hline $\begin{array}{l}\text { Culture in DMEM under } \\
\text { mammalian cell culture }\end{array}$ & Increase in number (Çiftçioglu et al 1997) & No increase in number and size \\
(Çiftçioglu et al 1997)
\end{tabular}

conditions

Size

Ultramicroscopic morphology

Chelation with EDTA or acids

Antibiotic/chemotherapeutic effect on cultures

Metabolic labelling (S-methionine, uridine) Recognition by monoclonal antibody for CNP (8DI0) Infectivity

Adaptation to physiological condition (morphological changes with protein concentration, antibiotics, heat) Stainability with pico-green, ribo-green, and Hoechst When injected intravenously to the rabbits

Polarized light treatment
Very narrow range (Kajander et al 1997;

Çiftçioglu et al 1997)

S-CNP: 80-500 nm

SF-CNP: $0.5-10 \mu \mathrm{m}$

Always have closed membranous vesicles involved with budding-like or septa-like formations

(Kajander et al 1997; Çiftçioglu et al 1997)

Release and precipitation of organic matter (Çiftçioglu and Kajander 1999; Kumar et al 2006) Sensitive to tetracycline, aminoglycosides, nucleic acid synthesis inhibitors, bisphosphonates, etc (Çiftçioglu et al 2002; Kajander et al 2003; Kumar et al 2006)
(+) (Kajander et al 1997; Miller et al 2004)
(+) (Çiftçioglu and Kajander 1998)
+ (Increased antibody level) (Çiftçioglu et al 2007)
(+) (Bjorklund et al 1998; Çiftçioglu and Kajander 1999; Çiftçioglu et al 2002, 2005)

(+) (Kumar et al 2006)

Goes to kidney (Akerman et al 1997)

Reduces biofilm formation (Sommer et al 2002)
Very wide range

$2 \mathrm{~nm}$ to centimeters or more

No "cell like" structure

Dissolves totally without any residue (Kumar et al 2006)

No effect (Kajander et al 2003;

Çiftçioglu et al 2002; Kumar et al 2006)

$(-)$

$(-)$

- No immune response

No effect (Çiftçioglu and Kajander 1999; Kumar et al 2006)

$(-)$

No specific organ (Donaldson and stone 2004)

No response (Kajander et al 2003; Sommer et al 2002)

Abbreviations: 
they are self-propagating (Angert 2006). Figures 3 and 7 are electron microscopic images of CNP showing both fission and budding-like, division-like formations.

The metabolic potential of CNP was confirmed using a tetrazolium salt detecting dehydrogenase activity, and S-methionine incorporation (Kajander et al 1997; Kumar et al 2006). Also, ß-mercaptoethanol, known to enhance growth of certain microorganisms and mammalian cells, promoted CNP metabolism and growth (Kumar et al 2006). Polarized light was shown to reduce CNP biofilm formation indicating a light induced metabolic process within CNP (Sommer et al 2002). Apparently CNP have metabolic activity which clearly differentiates them from inorganic crystal formation. It is obvious that these CNP manifest various functions besides self-replication, using an unidentified set of instructions originating from within their system.

Regardless of all the peer reviewed and published scientific literature related to CNP growth, antigenicity, infectivity and medical impact over the past 15 years, a controversy has continued to surge regarding the significance of CNP. However, it is also known that any uncharacterized nanoparticle in any biopharmaceutical product should be of concern. Nanoparticle toxicology concerns include translocation to the brain, general effects to the blood vessel wall and coronary artery disease (Donaldson 2006). CNP have been linked to many pathological calcification related diseases (Çiftçioglu et al 2006) and exert cytotoxic effects on some mammalian cells (Çiftçioglu and Kajander 1998; Akerman et al 1997). CNP are commonly present in bovine blood and thus in cell culture products, including vaccines and gammaglobulin (Çiftçioglu et al 1997). Our study was confined to pure CNP cultures. When CNP is present in blood circulation, they may bind protein and other blood components to their surface and increase the complexity of replication.

In the field of nanomedicine, nanoparticles are being developed for use in imaging and therapy (Freitas 2005). Medicinal nanoparticles are being designed similar to biological molecules so that they are biodegradable (Ebbesen and Jensen 2006). However, the very properties that make nanoparticles useful for new applications are also the very properties that make them harmful (SooChoi et al 2007). The surface charge of a nanoparticle determines as to what it can adsorb, penetrate, or adhere to (Nel at al 2006; Jallouli et al 2007; Ludlow 2007). CNP with their apatite envelope have been found to induce immune response against proteins adhered to them (Çiftçioglu et al 2007). Hence, caution must be exercised in the use of apatite nanoparticles or any other fine grain particles for vaccination, drug delivery, or cosmetics.

\section{Conclusions}

In this study we demonstrated the self-propagation of CNP, originally discovered in biopharmaceutical products, and the only blood-borne, nanometer-sized particles that have been correlated with several pathological calcifications. It is possible for nanoparticles to pass into biological products, remain undetected, and cause major health problems.

\section{Acknowledgments}

This research was funded by Astrobiology (NASA, JSC). We thank Nikon Instruments Inc. for lending the BioStation IM to perform the time-lapse experiments, Dr. Olavi Kajander (Nanobac) for his valuable comments and Patrice Colbert (NASA, JSC) for her assistance in editing the text.

\section{References}

Akerman KK, Kuikka JT, Çiftçioglu N, et al. 1997. Radiolabeling and in vivo distribution of nanobacteria in rabbit. In: Hoover B (ed). Instruments, Methods, and Missions for the Investigation of Extraterrestrial Microorganisms. Proc SPIE, 3111:436-42.

Altundag K, Altundag O, Akyurek S, et al. 2006. Possible association between nanobacteria and malignant microcalcifications in breast cancer. Breast J, 12:287.

Angert E. 2006. Beyond binary fission: Some bacteria reproduce by alternative means. Microbe, 1:127-31.

Angert ER. 2005. Alternatives to binary fission in bacteria. Nat Rev Microbiol, 3:214-24.

Bjorklund M, Çiftçioglu N, Kajander EO. 1998. Extraordinary survival of nanobacteria under extreme conditions. In: Hoover RB (ed). Instruments, Methods, and Missions for Astrobiology. Proc SPIE, 3441:123-9.

Çiftçioglu N, Aho KM, McKay DS, et al. 2007. Are apatite nanoparticles safe? Lancet, 369:2078.

Çiftçioglu N, Bjorklund M, Kajander EO. 1998a. Stone formation and calcification by nanobacteria in human body. In: Hoover RB (ed). Instruments, Methods, and Missions for Astrobiology. Proc SPIE, 3441:105-11.

Çiftçioglu N, Çiftçioglu V, Vali H, et al. 1998b. Sedimentary rocks in our mouth: dental pulp stones made by nanobacteria. In: Hoover RB (ed). Instruments, Methods, and Missions for Astrobiology. Proc SPIE, 3441:130-6.

Çiftçioglu N, Haddad RS, Golden DC, et al. 2005. A potential cause for kidney stone formation during space flights: enhanced growth of nanobacteria in microgravity. Kidney Int, 67:483-91.

Çiftçioglu N, Kajander EO. 1998. Interaction of nanobacteria with cultured mammalian cells. Pathophysiology, 4:259-70.

Çiftçioglu N, Kajander EO. 1999. Growth factors for nanobacteria. In: Hoover RB (ed). Instruments, Methods, and Missions for Astrobiology II. Proc SPIE, 3755:113-19.

Çiftçioglu N, Kuronen I, Akerman K, et al. 1997a. A new potential threat in antigen and antibody products: Nanobacteria. In: Brown F, Burton D, Doherty P, et al. (eds). Vaccines97. Cold Spring Harbor (NY): Cold Spring Harbor Laboratory Pr, pp. 99-103.

Çiftçioglu N, McKay DS, Mathew G, et al. 2006. Nanobacteria: Fact or fiction? Characteristics, detection and medical importance of novel selfreplicating, calcifying nanoparticles. J Investig Med, 54:385-94. 
Çiftçioglu N, Miller-Hjelle MA, Hjelle JT, et al. 2002. Inhibition of nanobacteria by antimicrobial drugs as measured by a modified microdilution method. Antimicrob Agents Chemother, 46:2077-86.

Çiftçioglu N, Pelttari A, Kajander EO, et al. 1997b. Extraordinary growth phases of nanobacteria isolated from mammalian blood. In: Hoover RB (ed). Instruments, Methods, and Missions for the Investigation of Extraterrestrial Microorganisms. Proc SPIE, 3111:429-35.

Cisar JO, Xu DQ, Thompson J, et al. 2000. An alternative interpretation of nanobacteria-induced biomineralization. Proc Natl Acad Sci, USA, 97:11511-15.

Donaldson K. 2006. Resolving the nanoparticle paradox. Nanomedicine, 1:229-34.

Donaldson K, Stone V. 2004. Nanoscience fact versus fiction. Commun ACM, 47:113-15.

Ebbesen M, Jensen TG. 2006. Nanomedicine: techniques, potentials and ethical implications. J Biomed Biotechnol, 5:1-11.

Forsythe E, Ewing F, Pusey M. 1994. Studies on tetragonal lysozyme crystal growth rate. Acta Cryst, D50:614-19.

Freitas Jr RA. 2005. Nnaotechnology, nanomedicine and nanosurgery. Int $J$ Surg, 3:243-6.

Freitas Jr RA, Merkle RC. 2004. Kinematic self-replicating machines. Georgetown, TX: Landes Bioscience.

Garnett J, Dieppe P. 1990. The effects of serum and human albumin on calcium hydroxyapatite crystal growth. Biochem J, 266:863-8.

Goe G. 1976. L-glutamic acid crystals grown from saturated aqueous solutions at $25^{\circ}$ and $37^{\circ} \mathrm{C}$. Mikrochim Acta, 66:119-34.

Jallouli Y, Paillard A, Chang J, et al. 2007. Influence of surface charge and inner composition of porous nanoparticles to cross blood-brain barrier in vitro. Int J Pharm, 344(1-2):103-9.

Kajander EO. 2006. Nanobacteria-propagating calcifying nanoparticles. Lett Appl Microbiol, 42:549-52.

Kajander EO, Bjorklund M, Çiftçioglu N, et al. 1999. Nanobacteria and man In: Seckcbach J (ed). Enigmatic Micro-organisms and Life in Extreme Environmental Habitats: Kluwer (the Netherlands). pp. 195-204.

Kajander EO, Çiftçioglu N. 1998. Nanobacteria: An alternative mechanism for pathogenic and intra- and extracellular calcification and stone formation. Proc Natl Acad Sci USA, 95:8274-9.

Kajander EO, Çiftçioglu N, Aho K, et al. 2003. Characteristics of nanobacteria and their possible role in stone formation. Urol Res, 31:47-54.

Kajander EO, Çiftçioglu N, Miller-Hjelle MA, et al. 2001. Nanobacteria: controversial pathogens in nephrolithiasis and polycystic kidney disease. Curr Opin Nephrol Hypertens, 10:445-52.
Kajander EO, Kuronen I, Akerman K, et al. 1997. Nanobacteria from blood, the smallest culturable autonomously replicating agent on earth. In: Hoover RB (ed). Instruments, Methods, and Missions for Astrobiology. Proc SPIE, 3111:420-8.

Kumar V, Farell G, Yu S, et al. 2006. Cell biology of pathologic renal calcification: contribution of crystal transcytosis, cell-mediated calcification, and nanoparticles. J Investig Med, 54:412-24.

Ludlow K. 2007. One-size fits all? Australian regulation of nanopraticle exposure in the workplace. $J$ Law Med, 15:136-52.

Malkin AJ, Kuznetsov YG, McPherson A. 1996. Incorporation of microcrystals by growing protein and virus crystals. Proteins, 24:247-52.

Miller-Hjelle MA, Hjelle JT, Çiftçioglu N, et al. 2003. Nanobacteria: Methods for growth and identification of this recently discovered calciferous agent. In: Olson WP (ed). Rapid Analytical Microbiology. Godalming, Surrey, UK: Davis Horwood International Publishing, Ltd., pp. 297-312.

Miller VM, Rodgers G, Charlesworth JA, et al. 2004. Evidence of nanobacterial-like structures in calcified human arteries and cardiac valves. Am J Physiol Heart Circ Physiol, 287:1115-24.

Nel A, Xia T, Madler L, et al. 2006. Toxic potential of materials at the nanolevel. Science, 311(5761):622-7.

Piana S, Reyhani M, Gale JD. 2005. Simulating micrometre-scale crystal growth from solution. Nature, 483:70-3.

Puskas LG, Tiszlavicz L, Razga Z, et al. 2005. Detection of nanobacterialike particles in human atherosclerotic plaques. Acta Biol Hung, 56:233-45.

Shiekh FA, Khuller M, Singh SK. 2006. Lithogenesis: induction of renal calcifications by nanobacteria. Urol Res, 34:53-7.

Sommer AP, Hassinen HI, Kajander EO. 2002. Light induced replication of nanobacteria: A preliminary report. J Clin Laser Med Surg, 20:241-4.

Soo Choi H, Liu W, Misra P, et al. 2007. Renal clearance of quantum dots. Nat Biotechnol, 10:1165-70.

Vali H, McKee MD, Çiftçioglu N, et al. 2001. Nanoforms: A new type of protein-associated mineralization. Geochim Cosmochim Acta, 65:63-74.

Wang L, Shen W, Wen J, et al. 2006. An animal model of black pigment gallstones caused by nanobacteria. Dig Dis Sci, 51:1126-32.

Wood HM, Shoskes DA. 2006. The role of Nanobacteria in urologic disease. World J Urol, 24:51-4. 
